\title{
Managing Depression Among Ethnic Communities: A Qualitative Study
}

\author{
Jobn Furler, MBBS, FRACGP, Grad \\ Dip, $P b D^{1}$ \\ Renata Kokanovic, $\mathrm{PbD}^{1}$ \\ Cbristopher Dowrick, MD, FRCGP2 \\ Danielle Newton, Grad Dip, $P b D^{1}$ \\ Jane Gunn, FRACGP, DRANZCOG, \\ $\mathrm{PbD}^{1}$ \\ Carl May, PbD, AcSS 3 \\ 'Primary Care Research Unit, Department \\ of General Practice, The University of Mel- \\ bourne, Australia \\ ${ }^{2}$ Division of Primary Care, The University \\ of Liverpool, Liverpool, United Kingdom \\ ${ }^{3}$ Institute of Health and Society, Newcastle \\ University, Newcastle-upon-Tyne, United \\ Kingdom
}

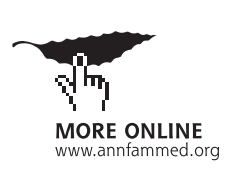

Conflicts of interest: none reported

\author{
CORRESPONDING AUTHOR \\ John Furler MBBS, FRACGP, \\ Grad Dip, PhD \\ Primary Care Research Unit \\ Department of General Practice \\ The University of Melbourne \\ 200 Berkeley St, Carlton, Victoria 3053 \\ j.furler@unimelb.edu.au
}

\begin{abstract}
PURPOSE Clinical care for depression in primary care negotiates a path between contrasting views of depression as a universal natural phenomenon and as a socially constructed category. This study explores the complexities of this work through a study of how family physicians experience working with different ethnic minority communities in recognizing, understanding, and caring for patients with depression.
\end{abstract}

METHODS We undertook an analysis of in-depth interviews with 8 family physicians who had extensive experience in depression care in 3 refugee patient groups in metropolitan Victoria and Tasmania, Australia.

RESULTS Although different cultural beliefs about depression were acknowledged, the physicians saw these beliefs as deeply rooted in the recent historical and social context of patients from these communities. Traumatic refugee experiences, dislocation, and isolation affected the whole of communities, as well as individuals. Physicians nevertheless often offered medication simply because of the impossibility of addressing structural issues. Interpreters were critical to the work of depression care, but their involvement highlighted that much of this clinical work lies beyond words.

CONCLUSIONS The family physicians perceived working across cultural differences, working with biomedical and social models of depression, and working at both community and individual levels, not as a barrier to providing high-quality depression care, but rather as a central element of that care. Negotiating the phenomenon rather than diagnosing depression may be an important way that family physicians continue to work with multiple, contested views of emotional distress. Future observational research could more clearly characterize and measure the process of negotiation and explore its effect on outcomes.

Ann Fam Med 2010;8:231-236. 10.1370/afm.1091.

\section{INTRODUCTION}

$\mathrm{D}$ epression remains a problem for primary care practice and research. ${ }^{1}$ Some see depression as independent of time, place, and culture ${ }_{i}$ common in primary care $_{i}{ }^{2}$ and effectively managed there. ${ }^{3,4}$ In this view, research should focus on improving management through training doctors, providing public education, and reorganizing practice systems. ${ }^{5}$ Others suggest that concepts of depression are socially constructed, varying across time and culture ${ }^{6}$ with the medicalization of personal distress reflecting a social and political balance based on the needs and interests of a range of groups that includes the medical profession, pharmaceutical companies, ${ }^{7}$ and the patients and general population. In this view, research should focus on understanding how such a balance emerges and how the concept of depression is sustained. ${ }^{1}$

Management of depression in primary care must somehow negotiate a path through these diverse views. In so doing, it is shaped by the microinteractions between patient and doctor, and by professional and societal contexts. ${ }^{8}$ Although formal recognition of depression may make little dif- 
ference to ${ }^{9}$ and may even adversely affect ${ }^{10}$ long-term outcomes, depression survives as a diagnosis ${ }^{1}$ and remains a good enough concept to provide a foundation for the essential medical work of responding to suffering. In this view, the task of research is to grasp the complexities of this work. ${ }^{11}$

Working across cultural differences is commonplace in primary care. Culture and communication are particularly relevant in depression care, where dialogue is crucial to the therapeutic doctor-patient interaction. ${ }^{7}$ Some suggest depression is particularly underrecognized and undertreated among minority groups. ${ }^{12}$ Language and communication barriers, lack of access to bilingual health workers, and translated patient educational material are cited as reasons. ${ }^{13}$ Others note that culture frames notions of normality, shaping beliefs and responses to experience of illness. ${ }^{14,15}$ The health professionals' own culture shapes expectations of encounters with patients and clinical practice. ${ }^{16}$ Culture plays an important role in shaping the doctor-patient relationship, ${ }^{17-21}$ in which different values, beliefs, and preferences can create problems for effective depression care. ${ }^{22,23}$ Cross-cultural research can lead to a deeper understanding of the way patient and doctor manage depression care.

We report how family physicians experience working across cultures with patients from different ethnic minority communities in 2 metropolitan regions of Australia: how these physicians recognize depression in patients from these communities, how they understand the nature of depression in these patients, and how they negotiate depression care in this setting.

\section{METHODS}

This report derives from a cross-cultural study within a study of depression in Australian primary care (Reorder, Appendix 1). The cross-cultural study was designed by a multisite, multidisciplinary team of investigators representing academic family practice, medical sociology, and psychiatry. One team member (J.F.) worked part time in the Community Health Centre (CHC) within which part of the study was based.

The cross-cultural study involved 73 interviews with health and welfare professionals at sites in Victoria and Tasmania, selected for their experience in providing primary care to minority communities. The Victorian metropolitan CHC involved in the study is located in a housing estate in a socioeconomically deprived area. It provides health and welfare services, including refugee advocacy, individual and family counseling, and mental health programs. The $\mathrm{CHC}$ serves a high proportion of migrants and refugees, particularly East Timorese and Vietnamese, who range from recent arrivals to those who have been in Australia for more than 20 years. All CHC family physicians have extensive experience working with these communities. The family physicians recruited from Tasmania have experience working with a range of refugee and migrant communities in Tasmania. The physicians interviewed for this study, which include all the family physicians at the $\mathrm{CHC}$, are the subject of this report $(\mathrm{N}=8)$. Interviews lasted from 1 to 1.5 hours. All interviews were audio-recorded and transcribed.

In semistructured interviews undertaken by one of the investigators (R.K.) and a research assistant, we asked the physicians to recount stories of caring for patients from these ethnic communities who were experiencing symptoms of depression. Key areas covered included (1) the physician's own views of depression (concepts of depression and its causes, the diagnostic, treatment and management process); and (2) the physician's experiences of working in their particular setting with refugees and migrant communities providing care to patients experiencing depression.

The full interview schedule is in the Supplemental Appendix, available at http://annfammed.org/cgi/ content/full/8/3/231/DC1. Ethics approval for the study was granted by The University of Melbourne Human Research Ethics Committee.

\section{Analysis}

Iterative thematic analysis was conducted following Mays and Pope's ${ }^{25}$ and Pope's ${ }^{26}$ framework for qualitative research and analysis in health care. We developed concepts from the data, rather than using preconceived theoretical frameworks. Three authors (R.K., J.F. and D.N.) read transcripts and analyzed them independently to identify themes and categories. Results were compared and discrepancies discussed with the wider group, and concepts were further refined. Additional thematic categories were added as the analysis developed. Transparency in analysis and reporting was achieved by providing extensive verbatim quotes and independent assessments of transcripts and themes. ${ }^{27}$

\section{RESULTS}

Demographic details of the physicians is displayed in Table 1 . None of the physicians interviewed had the same cultural or language as the communities being considered here; all spoke English as their first language.

Themes emerged from across the data from interviews with the family physicians working with all of the communities involved. Individually and together they provide insight into the way depression emerges as an object of work between doctor and patient. 
Table 1. Family Physician Demographic Information

\begin{tabular}{|c|c|c|c|c|c|c|c|c|}
\hline Characteristic & $\begin{array}{c}\text { Physician } \\
1\end{array}$ & $\begin{array}{c}\text { Physician } \\
5\end{array}$ & $\begin{array}{c}\text { Physician } \\
9\end{array}$ & $\begin{array}{c}\text { Physician } \\
11\end{array}$ & $\begin{array}{c}\text { Physician } \\
19\end{array}$ & $\begin{array}{c}\text { Physician } \\
33\end{array}$ & $\begin{array}{c}\text { Physician } \\
44\end{array}$ & $\begin{array}{c}\text { Physician } \\
88\end{array}$ \\
\hline Sex & Male & Male & Female & Female & Male & Male & Female & Male \\
\hline Age & 50 & 55 & 55 & 47 & 40 & 42 & 35 & 69 \\
\hline Country of birth & Australia & Australia & Australia & Australia & Australia & Australia & Australia & Australia \\
\hline $\begin{array}{l}\text { Languages other } \\
\text { than English } \\
\text { spoken }\end{array}$ & None & French & $\begin{array}{l}\text { Italian, } \\
\text { Spanish }\end{array}$ & Hebrew & Italian & None & None & None \\
\hline $\begin{array}{l}\text { Length of time in } \\
\text { profession, } y\end{array}$ & 19 & 27 & 31 & - & 10 & 12 & 0.5 & 42 \\
\hline $\begin{array}{l}\text { Length of time } \\
\text { working with this } \\
\text { community, y }\end{array}$ & 12 & 25 & 24 & - & 10 & 7 & 0.5 & 7 \\
\hline $\begin{array}{l}\text { Ethnic minority } \\
\text { population pri- } \\
\text { marily seen at } \\
\text { regular practice }\end{array}$ & $\begin{array}{c}\text { East } \\
\text { Timorese and } \\
\text { Vietnamese }\end{array}$ & $\begin{array}{c}\text { East } \\
\text { Timorese and } \\
\text { Vietnamese }\end{array}$ & $\begin{array}{c}\text { East } \\
\text { Timorese and } \\
\text { Vietnamese }\end{array}$ & $\begin{array}{c}\text { East } \\
\text { Timorese and } \\
\text { Vietnamese }\end{array}$ & $\begin{array}{c}\text { East } \\
\text { Timorese and } \\
\text { Vietnamese }\end{array}$ & Sudanese & Sudanese & Sudanese \\
\hline
\end{tabular}

\section{Understanding and Negotiating the Problem of Depression}

The physicians believed that patients from refugee communities often had difficulty with Western biopsychiatric concepts of depression. Sadness, worry, and stress were more commonly used terms, but reaching an agreed-upon understanding between doctor and patient about the nature of symptoms being experienced and what should or could be done was a challenge:

I think most people understand sadness...but in some cultures, they don't understand [depression] as a condition that requires treatment (Physician 9).

Agreement about the phenomenon being discussed was approached tangentially, in a slow process that required sensitivity:

So to broach issues around mental health you need more euphemisms: worry, maybe sadness, stress, anxiety $;$ you need to tiptoe...tread more softly, softly... because I think it [depression] is not a concept that they'd really relate to (Physician 11).

The physicians described these patients as reluctant to view their condition as an individual issue, as something happening to them. Focusing on community and contextual factors, such as family, work, financial, and housing issues, was seen as more acceptable. The physicians avoided the term depression during these discussions:

$[\mathrm{P}]$ eople are not really happy to discuss depression in terms of, like, it's a personal change that has happened in me...you are more likely to keep the discussion going about family, work, or housing or money, stuff like that (Physician 1).

The physicians believed these patients saw the physician's role as addressing physical problems, not knowing or accepting that a physician could also help with emotional distress:
[T] hey see you as a "treater" primarily of physical problems that is the model they have inside their head, so why would they...tell the doctor about all this sort of stuff, because what can they [the doctor] do...in fact they don't realize that telling is the part of the cure, (laughs)... a large part (Physician 5).

Perceptions of power and authority were seen as presenting difficulties in allowing the sort of dialogue needed to negotiate the problem:

I have a sense that...there's a bit of the doctor-is-God type of view, that if the doctor says this is what ought to happen ... [there is] perhaps a reluctance...to question my diagnosis or management... and so the management might not always be optimal (Physician 33).

The physicians thus saw the process of dialog, exchange, and negotiation as an important element of the work of depression care.

\section{Managing the Depression}

Refugee and settlement experiences loomed large. Prolonged experience of violence, together with the associated social isolation, language barriers, disconnectedness, unemployment, and family disintegration, were seen as common underlying causes of depression. The larger contextual framework of suffering for some physicians blurred boundaries between emotional distress and clinical depression:

I suspect there are a whole lot of people out there, because of awful trauma in their lives, awful events, (who) have times of deep sorrow, which is really what depression is, and sometimes people are quite desperate about it all (Physician 44).

Isolation compounded the effects of past trauma. Patients would worry about the safety of family who remained in the patient's country of origin: 
The other issue... is just lack of social supports within the community particularly their family... the family is split... some are in (the) country of origin and some are here, and all of the complications to their lives and stress that that creates... (Physician 5).

This contextual framing meant that the physician understood the condition being managed not only in terms of the individual patient's life, but as a problem at a community level:

I think that there's a challenge...they haven't got the supports around them... how do you support that community to form new links that replace the older links or, ah..., or reinforce the older ones but in a different way (Physician 9).

The physicians felt a need to do something for individual patients. Faced with the impossibility of addressing structural issues, to work around the barriers posed by complexities of communication via interpreters, their main recourse was to prescribe an antidepressant medication:

I just think that a doctor sitting there deciding what to do for this patient, if their communication is greatly impaired because of language barriers, they want to do something, so they are more likely to use medication, so I think that's just an accepted reality. ... I don't think it is a good thing, but it is how we try and manage in a multicultural society (Physician 5).

Yet understanding symptoms in the particular traumatic context of these communities meant that expectations of success with medication could be low:

I'd always try it [antidepressants]. But you have to be prepared for the fact that the damage that's been done is so severe that it will never be quite right again (Physician 44).

Thus although the physicians talked about these patients as experiencing depression, they drew simultaneously on historical, cultural, contextual, and medical understandings of the condition. They had to work with the patient's own understanding of their symptoms in a process of negotiation.

\section{Working With the Interpreter}

Physicians in the study had to use interpreters to work with patients from these communities to explore the painful experiences of trauma, torture, and social isolation. As passive conduits of words and information, interpreters could put this work at risk. Ideally interpreters had to be skilled in working beyond words:

[A] key point is having an interpreter who can convey the importance of emotional empathy... so if you slow the consultation down and speak softly, and use open words, trying to get the patient to emotionally release a bit, so the interpreter needs to be really good at that...you pick up cues from the interpreter about the emotional content of what is being interpreted. So that's important, so it is not just the words, you can work with interpreters who are just simply doing the words and that can be counterproductive.. (Physician 1)

Sometimes interpreters had a direct and important connection to patients:

I sometimes think it is actually their [interpreters'] manner and their demeanor in the consultation which is as much benefit as the questions that I'm feeding them, because in the end the patient is mostly looking at them when they are answering the question, so the response the interpreter has of the nodding and the acknowledgment and the way they ask the questions and the way they receive the answers really are quite critical in...that sort of therapy... (Physician 5).

The relationship between doctor and interpreter thus became an important factor in the therapeutic encounter with a patient:

$[\mathrm{T}]$ here is that issue of trusting the interpreter, trusting their professionalism, but also building a rapport, a relationship with the interpreter so that you have that confidence that they'll be translating what you're saying and giving you appropriate feedback.... [F]inding the right interpreters can sometimes be the answer (Physician 9).

The physicians were aware that this way of working with interpreters went beyond accepted norms of both medical and interpreting professions, bound by an ideal of interpreter as unengaged in the relationship between doctor and patient. ${ }^{28}$

\section{DISCUSSION}

The family physicians in this study occupied a borderland between several cultures. They described engaging with patients across differences in ethnicity and language in formulating the problem, the object of their work with patients. These interviews, however, also highlighted the professional culture across which these doctors worked, with clear diagnostic criteria for depression, notions of linguistic equivalence, and the physician as technical resource and care coordinator. They described their work as the act of negotiation across these worlds by means of a sensitive, implicit process. There were challenges. In their patients they saw traumatic life experiences; dislocation from community and social networks; fluid ideas of sadness, troubles, and worry rather than symptoms of depression; and the importance of sharing meaning and connection. Negotiation seemed inimical to these patients, who saw doctors as powerful figures of authority.

Interpreters could compromise the work of depression care if they worked simply to task; yet these interpret- 
ers were able, if skilled, to enhance depression care, connecting doctor and patient beyond words. The doctors were conscious of a need to do something, hence the drift toward labeling and diagnosis. Drawing on biomedical understandings of depression, they acknowledged its limitations.

Previous studies of physicians' experiences of depression care have reported different perceptions of the contributors to patients' depression, and family physician's subsequent approach to treatment. ${ }^{13,14}$ The physicians in our study also described a similar response. Rather than seeing these different worlds, cultures, and explanatory models as barriers to detection and management of depression or causes of poor compliance, however, physicians in our study saw negotiating these areas as integral to doing depression work in this context. They described enacting a particular form of depression care in primary care.

\section{Strengths and Limitations}

This study involved a small, unrepresentative sample of family physicians. The physicians caring for other cultural groups framed by different historical and contextual influences may report different experiences. It nevertheless provides a case study of depression care by physicians with rich experience of working across cultures. Although patient and interpreter views are reported in detail elsewhere, ${ }^{29}$ our report highlights the way experienced physicians may engage with cultural difference as an active element of depression care.

We asked the physicians in this study to choose patients to discuss who had achieved a diagnosis of depression at least at some level. In other words these patients had been subsumed within the medicalizing impulse ${ }^{29}$ of current depression work. The physicians may have been prompted to describe experiences of caring for depression that highlighted the importance of culture rather than seeing it as simply one of many influences. Previous research has shown that cultural difference is invoked in explaining difficulties and problems in the clinical encounter. ${ }^{30}$ Our study, however, suggests it may also be framed as an opportunity.

\section{Implications for Future Research and Clinical Practice}

Our findings highlight the need for more detailed observational research of clinical care for depression across a range of primary care settings and contexts. We do not know whether the family physicians in this study work similarly with patients who are not so obviously different. If they do, it would suggest that they continue to adopt a biomedical understanding of depression, finding it an efficient way to manage the work, while not questioning the underlying construct.
Neither do we know whether the physicians similarly approach patients from these communities who are experiencing emotional distress but are not given a diagnosis of depression. It is possible that the physicians support the notion of culturally flexible modes of expression of a range of emotional distress where, at times, the medicalizing notion of depression can usefully be used and others times not.

Our findings suggest a substantial vein of care for depression that would not necessarily be found through conventional studies of medical records, billing records, or patient self-report. Although it is possible that implicit, unspoken frameworks around depression care limit ways in which patients feel they can present symptoms legitimately, ${ }^{31}$ it suggests that depression care by family physicians occupies a unique form, and that grappling with difference is an important element of this work. Future research should better characterize and measure dimensions of this negotiating process as an active element of depression care and explore its relationship to clinical outcomes and physician and patient characteristics.

\section{Appendix 1. The Re-order Study and the Associated Cross-Cultural Study}

Re-order study. This study aims to reexamine how people experiencing depression are cared for in the community. The Re-order study draws together consumer views (computer-assisted telephone interviews with 576 people experiencing depression) and stakeholder views (a Delphi consultation with more than 300 practitioners, academics, and policy makers) exploring how family medicine should best meet the needs of people experiencing depression. The final practice phase of the study involves using both of these data sources in working with 6 family medicine practices to develop locally adapted best practice models of depression care.

Cross-cultural side study to Re-order. Designed to complement the main Re-order study and enhance the understanding of cultural perspectives in treating depression in primary care settings, it involved 73 in-depth qualitative interviews with patients from the Vietnamese, East Timorese, and Sudanese communities with family doctors and health care workers who were providing depression care to these communities.

Note: Re-order is a 3-year study by the Australian Primary Health Care Research Institute to investigate the management of depression in general practice, conducted in partnership with a team of academics from Australia and the United Kingdom. ${ }^{24}$

To read or post commentaries in response to this article, see it online at http://www.annfammed.org/cgi/content/full/8/3/231.

Key words: Minority health; depression; primary health care; cultural competency

Submitted January 26, 2009; submitted, revised, July 21, 2009; accepted August 31, 2009.

Funding support: This study was supported by a grant from the Australian Primary Health Care Research Institute (APHCRI), which is supported by the Australian Government Department of Health and Ageing under the Primary Health Care Research, Evaluation and Development Strategy. 
Disclaimer: The funding body did not have a role in study design; the collection, analysis, and interpretation of data; the writing of the manuscript; or the decision to submit this manuscript for publication. The information and opinions contained in it do not necessarily reflect the views or policies of the department.

Acknowledgments: We acknowledge the dedicated family physicians, their patients, and practice staff for making this research possible.

\section{References}

1. Pilgrim D. The survival of psychiatric diagnosis. Soc Sci Med. 2007; 65(3):536-547.

2. Goldman LS, Nielsen NH, Champion HC. Awareness, diagnosis, and treatment of depression. J Gen Intern Med. 1999;14(9):569-580

3. de Gruy F III. Depression research in primary care: pushing the field forward. Ann Fam Med. 2005;3(1):3-6.

4. Nutting PA, Gallagher K, Riley K, et al. Care management for depression in primary care practice: findings from the RESPECTDepression trial. Ann Fam Med. 2008;6(1):30-37.

5. Egede LE. Failure to recognize depression in primary care: issues and challenges. J Gen Intern Med. 2007;22(5)701-703.

6. Lee S. Diagnosis postponed: shenjing shuairuo and the transformation of psychiatry in post-mao China. Cult Med Psychiatry. 1999;23(3):349-380, discussion 381-399.

7. Dowrick C. Beyond Depression: A New Approach to Understanding and Management. Oxford: Oxford University Press; 2004.

8. Wittink MN, Barg FK, Gallo J]. Unwritten rules of talking to doctors about depression: integrating qualitative and quantitative methods. Ann Fam Med. 2006;4(4):302-309.

9. Dowrick C, Buchan I. Twelve month outcome of depression in general practice: does detection or disclosure make a difference? BMJ. 1995;311(7015):1274-1276

10. Wittkampf KA, van Zwieten M, Smits FT, Schene AH, Huyser J, van Weert HC. Patients' view on screening for depression in general practice. Fam Pract. 2008;25(6):438-444.

11. Robinson WD, Priest LA, Susman JL, Rouse J, Crabtree BF. Technician, friend, detective, and healer: family physicians' responses to emotional distress. J Fam Pract. 2001;50(10):864-870

12. Chung H, Teresi J, Guarnaccia P, et al. Depressive symptoms and psychiatric distress in low income Asian and Latino primary care patients: prevalence and recognition. Community Ment Health J. 2003:39(1):33-46.

13. Klimidis S, Minas H, Kokanovic R. Ethnic minority community patients and the Better Outcomes in Mental Health Care initiative. Australas Psychiatry. 2006;14(2):212-215.

14. Karasz A. Cultural differences in conceptual models of depression. Soc Sci Med. 2005;60(7):1625-1635.
15. Karasz A, Watkins L. Conceptual models of treatment in depressed Hispanic patients. Ann Fam Med. 2006;4(6):527-533.

16. Lupton D. Medicine as Culture: Illness, Disease and the Body in Western Societies. 2nd ed. London: Sage Publications; 2003.

17. Harmsen $H$, Meeuwesen L, van Wieringen J, Bernsen R, Bruijnzeels M. When cultures meet in general practice: intercultural differences between GPs and parents of child patients. Patient Educ Couns. 2003;51(2):99-106.

18. Laveist TA, Nuru-Jeter A. Is doctor-patient race concordance associated with greater satisfaction with care? J Health Soc Behav. 2002;43(3):296-306

19. Murray-García JL, Selby JV, Schmittdiel J, Grumbach K, Quesenberry CP Jr. Racial and ethnic differences in a patient survey: patients' values, ratings, and reports regarding physician primary care performance in a large health maintenance organization. Med Care. 2000;38(3):300-310.

20. Penn NE, Kar S, Kramer J, Skinner J, Zambrana RE. Ethnic minorities, health care systems, and behavior. Health Psychol. 1995;14(7): 641-646.

21. Ramirez AG. Consumer-provider communication research with special populations. Patient Educ Couns. 2003;50(1):51-54.

22. Cornford CS, Hill A, Reilly J. How patients with depressive symptoms view their condition: a qualitative study. Fam Pract. 2007;24(4) 358-364.

23. Rogers A, May C, Oliver D. Experiencing depression, experiencing the depressed: The separate worlds of patients and doctors. J Ment Health. 2001;10(3):317-333.

24. Palmer V, Gunn J, Kokanovic R, et al. Diverse voices, simple desires: a conceptual design for primary care to respond to depression and related disorders. Fam Pract. 2010; Apr 8 [Epub ahead of print].

25. Mays N, Pope C. Rigour and qualitative research. BMJ. 1995;311 (6997):109-112.

26. Pope C, Mays N. Reaching the parts other methods cannot reach: an introduction to qualitative methods in health and health services research. BMJ. 1995;311(6996):42-45

27. Yardley L. Dilemmas in qualitative health research. Psychol Health. 2000;15(2):215-228.

28. Hsieh $\mathrm{E}$. Conflicts in how interpreters manage their roles in provider-patient interactions. Soc Sci Med. 2006;62(3):721-730.

29. Kokanovic R, May C, Newton D, Dowrick C, Furler J, Gunn J. Negotiations of distress between East Timorese and Vietnamese refugees and their family doctors in Melbourne. Sociol Health IIIn. In press.

30. Wachtler C, Brorsson A, Troein M. Meeting and treating cultural difference in primary care: a qualitative interview study. Fam Pract. 2006;23(1):111-115

31. Baik SY, Bowers BJ, Oakley LD, Susman JL. The recognition of depression: the primary care clinician's perspective. Ann Fam Med. 2005;3(1):31-37. 